\title{
Les paradoxes d'un poète: Piron et la postérité
}

\section{Stéphanie Loubère}

\section{(2) OpenEdition}

\section{Journals}

\section{Édition électronique}

URL : http://journals.openedition.org/studifrancesi/9763

DOI : 10.4000/studifrancesi.9763

ISSN : 2427-5856

\section{Éditeur}

Rosenberg \& Sellier

\section{Édition imprimée}

Date de publication : 1 août 2017

Pagination : 236-248

ISSN : 0039-2944

\section{Référence électronique}

Stéphanie Loubère, "Les paradoxes d'un poète: Piron et la postérité », Studi Francesi [En ligne], 182 (LXI | II) | 2017, mis en ligne le 01 août 2018, consulté le 07 janvier 2021. URL : http:// journals.openedition.org/studifrancesi/9763 ; DOI : https://doi.org/ERREUR PDO dans /localdata/ www-bin/Core/Core/Db/Db.class.php L.34 : SQLSTATE[HY000] [2006] MySQL server has gone away

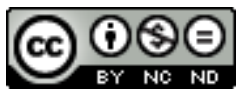

Studi Francesi è distribuita con Licenza Creative Commons Attribuzione - Non commerciale - Non opere derivate 4.0 Internazionale. 


\title{
Les paradoxes d'un poète: Piron et la postérité
}

\begin{abstract}
Once famous for his scandalous Ode to Priapus and quite forgotten thereafter, Piron is an author worth being read anew to gain an insight into how a poet could claim a place in the civil and literary society. Considering posterity with irony is a common demeanour among poets in his time. Some of them are aware that they are practising a fugitive form of art and self-mockery can merge with praise of their dignified vocation in their works. Resolute evanescence and wilful levity can lead to a complex conception of how they want to accede to or hide from posterity. Piron, as some other poets, yearns for an bic et nunc recognition, proclaiming: «Être c'est tout, avoir été n'est rien» (to be is everything, to have been is nothing). Still far from being a cursed poet, he cannot give up his desire to address the generations to come. Piron's work shows an obsessive concern for poetic glory, usually seen as unachievable, regardless of all the efforts the poet can make. Sometimes he names and shames the vain desire of posterity and sometimes he proudly asks for his right to achieve literary immortality. There is a paradox inherent in being a self-declared "light poet": conspicuous modesty does not prevent him from being conscious and proud of his talent. Piron is paradigmatic of this particular stance: arrogant humbleness is to be found throughout his work and makes him part of a poetical family that can be described by its will to reconcile an ideal of levity and an ambition to give literary dignity to a minor form of poetry. Still a young poet, Piron wrote an epitaph where he calls himself the «poète qui ne fut rien» (the poet who was nothing) only to end his life and career with an Apothéose de Binbin (his nickname), where he playfully claims for himself a well-deserved immortality. An accurate scrutiny of Piron's work, presenting such an ambiguous sample of attitudes towards posterity, could lead to a useful reassessment of the Enlightenment poetry and poetics.
\end{abstract}

Piron, si l'on se souvient encore de lui, est un poète au sens très large que le terme pouvait avoir en son siècle: les dix tomes de ses œuvres complètes rassemblent ses pièces pour la Foire, ses tragédies et comédies pour la Comédie Française, ses opéras comiques, mais aussi des contes galants, des poèmes allégoriques, des fables, des satires, des chansons, des épigrammes à foisons, beaucoup de pièces de circonstances et quelques poésies religieuses.

Originaire de Dijon, il embrasse la carrière des lettres et se fait connaître par le scandale de son Ode à Priape, dont le souvenir sulfureux lui fermera les portes de l'Académie Française. Il connaît quelques succès sur diverses scènes de la capitale, mais pour son siècle et celui qui suivra, il est surtout célèbre pour La Métromanie, une comédie de 1738 où il décline à peu près tous les visages que peut faire prendre la fureur de faire des vers aux poètes de son temps. La pièce est retombée dans l'oubli, et si son nom surnage après le grand déluge qui a englouti presque toute la poésie des Lumières après Sainte-Beuve, c'est le plus souvent d'une façon paradoxale. On cite volontiers et pour faire vite l'épitaphe qu'il composa lui-même:

Ci-git Piron qui ne fut rien,

Pas même académicien.

L'épisode de son rejet de l'Académie peut être rappelé pour comprendre tout le sel et le sens de cette épitaphe qui est à l'image du rapport particulier qu'il entretenait 
avec l'idée de sa survie et plus généralement avec celle de la mémoire qui est due aux poètes. Il est reçu à l'Académie Française en 1753 mais, sous la pression d'une cabale qui le dénonce comme l'auteur de la trop licencieuse Ode à Priape, Louis XV refuse de ratifier l'élection. Il n'aura de cesse par la suite de maltraiter les quarante immortels et leur institution nationale à grand renfort d'épigrammes moqueuses et de piques assassines. Paradoxalement, cette radiation sera pour lui l'occasion de passer à la postérité, puisque son épitaphe fit sans doute plus pour prolonger sa mémoire que ne l'aurait fait son entrée à l'Académie.

Le paradoxe est confirmé par la lecture de son œuvre, traversée par une réflexion sur l'immortalité que briguent parfois en vain les poètes et que décerne parfois à tort l'Académie, sur les mirages et les promesses de la postérité. Il fallut à Piron trouver un autre moyen que l'inscription dans les registres pour affirmer son droit à la mémoire collective: ce sera de n'être rien, «pas même académicien», avec panache et lucidité, morgue et modestie. Très tôt, avant même la désagréable expérience de son rejet de l'Académie, Piron adopte une posture ambivalente à l'égard de ses ambitions poétiques, qu'il soumet à une satire impitoyable sans jamais y renoncer. Pour lui la postérité est à la fois un objet de grande méfiance, de raillerie, et le point de fuite de toute sa réflexion sur le rôle et la place du poète dans la société civile et littéraire.

C'est dans cette oscillation que Piron trouve son originalité et gagne à être relu, pour mieux apprécier la complexité du rapport que les poètes de son siècle ont pu entretenir avec la postérité. J'évoquerai différents aspects de cette oscillation qui traverse toute son œuvre: sa représentation du poète, sa façon de concevoir la transmission d'une œuvre ou de prendre place dans l'histoire en tant que poète témoignent d'une conscience indocile, capable de passer le désir d'immortalité au crible d'une lucidité qui se révèle étonnamment moderne.

\section{Le parasite et le parangon}

L'œuvre polymorphe de Piron rend compte d'une incessante réflexion sur la

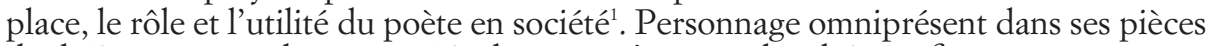
de théâtre comme dans sa poésie, le poète n'est pas chez lui une figure aux contours fixes: tantôt un type comique, tantôt l'incarnation d'un idéal, il permet à Piron de cerner les facettes parfois contradictoires de l'homme de génie.

On trouve de nombreux personnages de poètes ridicules dans son œuvre, notamment dans La Métromanie, qui en propose l'échantillon le plus varié. On peut rappeler que cette pièce est inspirée par un fait réel, une supercherie littéraire qui avait ridiculisé les admirateurs d'une supposée grande poétesse, en réalité un certain Desforges-Maillard ayant imaginé ce moyen de faire parler de lui². La comédie du petit monde littéraire offrait ainsi à la scène un caractère dont les travers prêtaient à rire: le personnage de Damis, jeune poète habité par son art, est d'abord présenté comme

(1) Cet aspect de l'œuvre de Piron est au centre de la thèse que lui consacra P. VerèB: Alexis Piron, poète (1689-1773) ou la difficile condition d'auteur sous Louis XIV, Oxford, SVEC, 1997.

(2) Sur l'affaire Antoinette Malcrais de La Vigne, voir la notice de J. TrucheT pour la Métromanie dans Théâtre du XVIII siècle, Paris, Gallimard, 1972, t. I, pp. 1456-1457. Ä partir de 1729 avaient paru dans le Mercure des poésies signées Antoinette Malcrais de la Vigne, présentée comme une demoiselle bretonne. Des admirateurs écrivirent au journal pour louer la poétesse, Voltaire même se laissa aller à lui écrire une épître où il vante sa «muse divine» (À Mademoiselle de Malcrais de la Vigne, du Croisic en Bretagne, «Mercure de France», mai 1732, pp. 917-920). Derrière ce pseudonyme se cachait en réalité Paul Desforges-Maillard qui avait réussi par ce biais à faire remarquer ses vers. La supercherie, une fois révélée, fit beaucoup rire, mais Voltaire resta un peu amer d'en avoir été la dupe. 
un «visionnaire» qui trébuche sur les pavés et vit dans le monde de ses chimères. Le portrait qu'en donne son valet Mondor au début de la pièce le situe d'emblée du côté des extravagants ridicules qui peuplent le monde comique:

Au surplus, rassemblant, dans sa seule personne,

Plusieurs originaux qu'au théâtre on nous donne:

Misanthrope, Étourdi, Complaisant, Glorieux,

Distrait... ce dernier-ci le désigne le mieux.

Et tiens, s'il est ici, je gage mes oreilles

Qu'il est dans quelque allée à bayer aux corneilles,

S'approchant, pas à pas, d'un ahah qui l'attend,

Et qu'il n'apercevra qu'en s'y précipitant ${ }^{3}$.

On trouve dans la pièce un autre spécimen de métromane comique en la personne de Francaleu, bourgeois respectable pris sur le tard par la folie des vers, qu'il fait «sans peine» ${ }^{4}$ et publie dans le Mercure sous le nom de «Mlle de Mériadec de Kersic de Quimper Corentin». Plus discret car n'apparaissant pas sur scène, il faudrait ajouter le père du jeune amant, qu'une missive en vers suffit à convaincre à laver un différend judiciaire et à donner son accord pour un mariage: il signe du nom de «Métrophile», oubliant ses intérêts matériels pour se réfugier dans le monde improbable où la loi positive s'effacerait devant la loi poétique.

Le poète n'est cependant pas qu'un ridicule de comédie pour Piron. Plusieurs anecdotes, rapportées par Rigoley de Juvigny dans sa Vie de Piron, témoignent de l'attachement de l'auteur à son titre de poète. Ainsi, après avoir passé une joyeuse soirée avec ses amis les poètes Gallet et Collé, Piron aurait été arrêté par la garde, et défendu sa cause dans un interrogatoire loufoque et exalté:

«Qui êtes-vous? votre nom?»- «Piron»-«Quel est votre état?»- «Poète»- «Poète?» - «Oui, monsieur, poète. Eh! où donc vivez-vous pour ne pas connaître le poète Piron? [...] Oui, monsieur, mon état est d'être poète, état le plus grand, le plus noble, le plus sublime que les hommes puissent embrasser, quand c'est du génie qu'ils le tiennent!»'.

Fier de son état de poète, il était capable d'en défendre crânement l'honneur si nécessaire:

Il s'était fait une si haute idée de l'état d'homme de lettres, qu'on ne doit point être étonné de la fierté avec laquelle il en soutenait la noblesse. Il ne souffrit jamais qu'on osât la rabaisser en sa présence; et c'est ce qu'il fit sentir à un grand seigneur, dans l'appartement duquel il était prêt d'entrer, au moment où celui-ci reconduisait une personne qualifiée. «Passez, Monsieur, dit le maître du logis à la personne qui s'arrêtait par politesse; passez, ce n'est qu'un poète» - «Puisque les qualités sont connues, répartit Piron, je prends mon rang», et il passa le premier.

Le plus souvent cependant, le poète ne se présente pas chez Piron de façon uniformément ridicule ou élogieuse. Dans La Métromanie, il est alternativement présenté

(3) A. Piron, La Métromanie, ou Le poète, dans Euvres complètes illustrées de Alexis Piron, éd. P. Dufay, Paris, Guillot, 1928-1931 (désormais OC), t. III, acte I, scène 1, p. 48.

(4) Ibid., acte II, scène 1 , p. 79.

(5) Vie d'Alexis Piron, dans Euvres complètes d'Alexis Piron, publiées par M. Rigoley de Juvigny, Paris, Lambert, 1776, tome I, pp. 74-75.

(6) Ibid., p. 117. 
comme un distrait de comédie, un fâcheux assommant son entourage par la lecture de ses vers, mais aussi comme un poète inspiré, conscient des sacrifices réclamés par son art, un cœur pur que rien ne détourne de son idéal et qui porte haut l'ambition de son génie ${ }^{7}$. Sa prétention à la gloire nourrit l'un des moments de la pièce où la satire se mêle subtilement à l'éloge, et où le spectateur est convié à rire de ce qu'il est par ailleurs forcé d'admirer:

Le nourrisson du Pinde, ainsi que le guerrier,

À tout l'or du Pérou préfère un beau laurier.

$[\ldots]$

Qu'on me laisse, à mon gré, n'aspirant qu'à la gloire,

Des titres du Parnasse anoblir ma mémoire,

$[\ldots]$

[Ma vertu] donc se borne au mépris des richesses,

Â chanter des héros de toutes les espèces,

À sauver, s'il se peut, par mes travaux constants,

Et leurs noms et le mien des injures du temps ${ }^{8}$.

Le portrait du poète dans la pièce est donc un portrait nuancé qui rejoint l'autoportrait ambivalent qui se détache des divers écrits de Piron: le poète y apparaît alternativement ou simultanément comme un parasite voué à disparaitre avec le milieu dans lequel il prospère ou comme un parangon offert aux générations futures. Surtout, Piron s'applique à lui-même ce regard mêlé, qui tient le milieu entre le poète sacré et le poète crotté. On le voit ainsi revendiquer «folie, gueuserie et vanité» comme «trois avantages de nature» qui sont aussi les «trois bases du noble métier des vers, sans lesquelles on passe tout doucement son chemin comme d'honnêtes gens, sans rats, sans faute et sans poux» ${ }^{9}$, et se présenter comme un «va-nu-pieds» sur «le maudit chemin $[\ldots .$.$] de la gloire»:$

Ah! monsieur, le maudit chemin que celui de la gloire! De jour en jour je définis la gloire un château en Espagne, où l'on arrive par une longue avenue tortueuse, pleine de brouillards, bordée de chimères, et pavée d'épines. Le beau diable de tapis pour un va-nu-pieds comme moi! mais que faire? Il n'est plus temps que je tourne la tête, pousse en avant, et haut le pied, pauvre Piron, tout vient à point à qui peut attendre ${ }^{10}$.

La conscience du «ridicule» de l'ambition poétique est récurrente chez lui. On la trouve formulée dans sa correspondance privée:

Il est ridicule de s'amuser à des travaux laborieux et nos travaux ne passent que pour des amusettes. L'orgueil poétique n'en convient pas. Mais notre mauvaise fortune devrait nous réduire à l'avouer, tout ce qui n'enrichit pas ne devrait occuper personne et sur ce principe, comment nommer nos occupations? ${ }^{11}$.

(7) Pour une analyse détaillée de la polarisation et de la diffraction du personnage du poète dans la pièce, voir S. Loubère, Piron ou l'apothéose du poète qui ne fut rien, «Lumen» 35, 2016, pp. 1-17.

(8) La Métromanie cit., acte III, scène 7, pp. 125-127.

(9) «J'ai, par dessus vous, trois grands avantages de nature: folie, gueuserie et vanité; trois bases du noble métier des vers, sans lesquelles on passe tout doucement son chemin comme d'honnêtes gens, sans rats, sans faute et sans poux; et je ne donnerais pas un fétu de qui s'embarque sur la mer d'Hippocrène, sans cette cargaison» (Lettre à l'abbé Legendre (7 novembre 1728), dans Alexis Piron épistolier, éd. G. von Proschwitz, «Romanica Gothoburgensia» 20, 1982, p. 68).

(10) Lettre à l'abbé Legendre (5 octobre 1730), ibid., p. 79.

(11) Lettre au marquis de Sénas d'Orgeval (30 novembre 1730), ibid., p. 81. 
Et à la fin de son Apothéose de Binbin (1770), il clôt un dialogue imaginaire sur sa vocation poétique engagé avec Pégase par ces mots: «Mais laissons là ma ridicule histoire» ${ }^{12}$.

Il est ainsi ouvertement le poète faute de mieux, vaguement fou, dont il esquisse le portrait sans concession dans la préface autobiographique jointe en 1764 à La Métromanie:

Je ne me donne que pour ce que je suis, que pour un de ces esprits trop ordinaires qui reçoivent le jour, non sous l'astre bénin dont l'influence est si rare, mais sous cet astre pestilentiel et non moins dominant qui fait qu'on a la fureur d'être poète, et souvent, qui pis est, celle encore de se le croire.

Je cédai donc à la force majeure: ainsi peut bien s'appeler cette manie qui fait ici tout à la fois l'excuse bonne ou mauvaise de l'auteur, et le titre de la pièce, et je lui cédai d'autant plus naturellement, qu'après tout le bien et le mal qu'elle m'a causé, je ne pouvais manquer d'avoir une vive démangeaison d'en dire tout le mal et le bien que j'en pense ${ }^{13}$.

Modestie et fierté s'entremêlent ainsi sans cesse, et vont au-delà du simple artifice rhétorique. En donnant régulièrement la parole dans ses écrits aux béotiens qui dénoncent la paresse et l'inutilité des poètes, Piron pose continûment la question de leur rôle dans la société: qu'ont-ils à offrir à leur époque et à léguer à la postérité? La réponse apportée par Piron à la question de la filiation poétique est loin d'être simple.

\section{Le legs et le larcin}

À son oncle qui lui promet la misère mais aussi l'opprobre et l'oubli s'il s'obstine à vouloir être poète, le Damis de La Métromanie oppose la certitude de servir à son temps et d'inscrire son nom dans l'histoire par l'exemple de ses écrits:

Je veux que la vertu plus que l'esprit y brille.

La mère en prescrira la lecture à sa fille ${ }^{14}$.

La prétention de Damis à offrir son œuvre en exemple aux générations à venir rejoint celle de Piron à voir son œuvre lui survivre, fût-ce par des voies détournées. Cette formule, tout spécialement, a donné lieu à une filiation parodique qui a participé à la survie du poète: elle est régulièrement détournée pour servir de caution morale à des ouvrages qui le sont peu. On la retrouve ainsi en 1777 dans la Bibliothèque des amants de S. Maréchal («L'amant en prescrira la lecture à sa mie» ${ }^{15}$ ), dans Les Fureurs utérines, un libelle anonyme de 1791 qui renverse la formule de façon suggestive («La mère en proscrira la lecture à sa fille» ${ }^{16}$ ), ou encore chez Sade qui en fait malicieusement l'épigraphe de sa Philosophie dans le boudoir (1795). Cette forme de survie irrévérencieuse aurait beaucoup plu sans doute à un auteur chez qui le souci de la postérité connaît des intermittences et de sérieuses mises en question: on le voit ainsi présenter son œuvre tantôt comme un legs destiné aux générations futures et

(12) L'Apothéose de Binbin, ou Voyage de l'Empyrée, OC, t. VIII, p. 335.

(13) OC, t. III, p. 11.

(14) La Métromanie cit., acte III, scène 7, p. 130.

(15) S. Maréchal, Bibliothèque des amants, Odes érotiques, Paris, Duchêne, s. d. [1777].

(16) Les Fureurs utérines de Marie-Antoinette, femme de Louis XVI, Au Manège et dans tous les Bordels de Paris, 1791. 
tantôt comme un larcin qui leur confisquerait la parcelle d'invention poétique qu'il s'est accaparée dans son œuvre.

Piron ne s'affranchit pas de tout rapport de transmission: à la postérité, il entend léguer une œuvre, qu'il s'efforce de constituer comme telle. Il supervise la publication de ses Euvres complètes en 1764 , publication «officielle», avec préfaces explicatives de ses pièces pour les remettre en contexte et en orienter la réception pour les lecteurs à venir. La désignation d'un éditeur testamentaire (Rigoley de Juvigny) témoigne aussi de son souci de contrôler cette transmission ${ }^{17}$. Dans la préface qu'il rédige en 1758 pour sa pièce L'École des Pères ou Les Fils ingrats, il se démarque de ses contemporains qui «du mépris des Anciens, [ont] passé délibérément à celui de la postérité» et regrette que l'on accuse de «Postéromanie» ceux qui, comme lui, «auraient encore en vue les races futures $»^{18}$. S'il dénonce volontiers la vanité des auteurs qui courent après la renommée, cela ne l'empêche pas d'afficher sa fierté de pouvoir «laisser un nom»:

Oui, monsieur, je laisse un nom, dussent en enrager mille fois les académies dont je ne suis point et tous les protecteurs et vice-protecteurs du monde. Car jeudi Gustave a fait pleurer un gros auditoire comme à sa première représentation; et hier samedi La Métromanie a fait encore plus rire. Je ne vois pas un académicien tout immortels qu'ils se disent qui ait ces deux billets d'entrée au temple de Mémoire et cela vaut bien pour le moins le prix que vous n'avez pas eu et la place qu'on me dénie, et que je n'ai ni demandée ni désirée ${ }^{19}$.

Piron est plus incisif dans ces propos que dans les très rapides Vers à la Postérité qu'il compose sur un air connu: le manque de talent a conduit trop d'auteurs de son époque à dénigrer les grands modèles du passé qu’ils ne pouvaient égaler. Les «Vers à la Postérité» de Piron sont une sorte de legs négatif, celui du mauvais exemple à ne surtout pas suivre:

Postérité, réformez-vous,

Sur les sottises de notre âge:

Riez, si nous fûmes des fous:

Mais n'en devenez que plus sage! ${ }^{20}$.

On le devine, c'est surtout la dimension déceptive de ce petit poème qui est intéressante: c'est ailleurs dans son œuvre qu'il faut aller chercher les traces d'un souci de la postérité qui malmène la notion même d'héritage et participe à sa recherche si particulière d'une identité poétique.

Au détour d'un dialogue de sa Métromanie, Piron énonce un rapport à la postérité extrêmement moderne: le «pillage d'avance». Dans la scène 7 de l'acte III (qui vaut à sa façon parfois ambiguë comme un manifeste poétique de Piron) Baliveau le Capitoul promet à son neveu Damis le sort pitoyable qui attend la majorité des poètes, oubliés par la gloire. La grande Querelle s'invite alors sur scène. À Baliveau qui affirme que les anciens ont tout dit et «Moissonnaient à leur aise où l'on glane aujourd'hui» ${ }^{21}$, Damis rétorque:

(17) Sur l'opiniâtreté avec laquelle Piron a mené cette entreprise éditoriale, voir P. VERÈB, Alexis Piron, poète cit., pp. 280-289.

(18) OC, t. I. pp. 15-16.

(19) Lettre à François-Jean d'Orceau, baron de Fontette (22 juillet 1754), Alexis Piron épistolier cit.,

p. 151. Gustave Wasa, tragédie historique de Piron, fut jouée pour la première fois en 1733.

(20) Vers à la Postérité, OC, t. IX, p. 138.

(21) La Métromanie cit., p. 127. 
Ils ont dit, il est vrai, presque tout ce qu'on pense.

Leurs écrits sont des vols qu'ils nous ont faits d'avance;

Mais le remède est simple: il faut faire comme eux;

Ils nous ont dérobés, dérobons nos neveux;

Et, tarissant la source où puise un beau délire,

À tous nos successeurs ne laissons rien à dire.

Un démon triomphant m'élève à cet emploi:

Malheur aux écrivains qui viendront après moi! ${ }^{22}$.

La satire du poète glorieux est inséparable ici d'une postulation qui permet d'échapper à l'agon parfois stérile de la Querelle. C'est évidemment une pirouette pour formuler de manière provocatrice un projet moral identifiable, l'ambition de se hisser au rang de modèle, mais le mot d'ordre violent et moderne de «dérober [les] neveux» (qui annoncerait presque les expérimentations décalées sur la réception des œuvres d'un Pierre Bayard ${ }^{23}$ !) invite à penser la création littéraire selon une logique différente, marquée par le refus de linéarité historique. En imaginant cette inversion du cours naturel des choses, où l'héritage ne transmet plus mais confisque, Piron donne à penser l'idée de filiation comme un enjeu complexe, contradictoire, où la création est réinstallée dans une temporalité qui la dégage des obligations dues à ce qui fut ou à ce qui viendra. C'est penser l'écriture comme un acte toujours moderne, quand la survie des œuvres et des auteurs, qui appartient à l'histoire, est sans cesse menacée de péremption.

\section{Vouloir être et aspirer au néant}

Cette dialectique de l'engendrement et de l'anéantissement est sans cesse présente dans l'œuvre de Piron. Dès 1722, dans sa pièce Arlequin Deucalion, il met en scène un personnage confronté à l'idée de la disparition de la vie humaine. Le déluge mythologique qui isole Deucalion au sommet du mont Parnasse et le contraint à soliloquer, permet à Piron de déjouer l'interdiction d'user de dialogues qui frappait alors le théâtre de la Foire. Contournant cet interdit en produisant une pièce monologue où s'élève la voix du dernier homme ayant survécu à la punition divine, Piron fait de son Arlequin le détenteur d'une parole menacée, et propose une vision de l'anéantissement total dans le poète de la poésie, lorsque son héros s'adresse à une Muse muette:

Que je meure sans lignée, adieu les hommes: plus d'hommes, plus de fous, donc plus de poètes: et qui vous cultivera dès lors? ${ }^{24}$.

La question de la postérité est posée à rebours de sa formulation habituelle, en mettant l'accent non pas sur le déploiement mais sur l'origine de la «lignée». En chaque homme, en chaque poète, se jouerait l'existence tout entière de l'humanité et de la poésie.

Piron pose ainsi un regard régulièrement décentré sur l'idée de survie et de transmission littéraires. Dans une lettre sur la Querelle adressée par d'«anciens Grecs»

(22) Ibid., p. 128.

(23) Voir P. BaYard, Le Plagiat par anticipation, Paris, Minuit, 2009 ou Et si les ceuvres changeaient d'auteur?, Paris, Minuit, 2010.

(24) Arlequin Deucalion, OC, t. IV, acte I, scène 3, p. 17. 
depuis le royaume de Pluton à un «jeune homme entêté des anciens, sans les avoir lus et sans avoir encore daigné lire un moderne» $(1716)^{25}$, la défense de la modernité prend le détour de l'ironie, puisqu'elle se donne à lire dans les conseils donnés par les anciens Grecs au jeune homme: pour l'inviter à apprendre une langue ancienne il s'appuient sur des citations en italien, langue vivante qui fait d'eux des «modernes malgré eux ${ }^{26}$. Surtout, les arguments des uns et des autres finissent par s'abolir: la lettre se clôt sur des vers anacréontiques que l'on peut lire comme une émanation directe de l'antique voix du poète grec ou comme son altération résolument moderne. Pour finir, Piron le poète signe sous le masque du philosophe Pyrrhon:

Le joyeux Anacréon vous envoie de ses nouvelles au pied de cette lettre. Quand vous saurez un jour lire ce gracieux auteur et que vous le relirez ici, vous aurez peine à le reconnaître. Écoutez donc, en deux ou trois mille ans de temps, on change bien: voyez déjà ce qu'il ressemble sous le nom de La Motte. Pour nous, nous serons constamment à vous.

Signés

Hésiode, Homère, Eschyle, Sophocle, Euripide, Hérodote, Anacréon, Ménandre, Aristophane et Pyrrhon ${ }^{27}$.

L'homonymie permet à la fois un effacement et une glorification: il appose son nom à peine camouflé à la fin d'une énumération d'auteurs anciens avec qui il partage bien des affinités et dont il se fait le contemporain, en même temps qu'il se démarque d'eux en avançant masqué. La dissimulation lui permet d'investir à la fois le présent (dans la lettre qui précède, sa voix se fait entendre derrière celle d'un nécromant «grand amateur de $[\mathrm{s}]$ on siècle» ${ }^{28}$ ) et le passé (avec cette signature détournée, qui le rapproche du philosophe sceptique). Pyrrhon, qui évite de donner un avis tranché et étend le doute sur tout, s'accorde bien avec Piron, capable de défendre les Modernes et de prendre sa place au milieu des Anciens, capable aussi de clamer sa fierté de poète et de s'effacer devant ses illustres modèles.

Piron ne prend pied dans son époque que par cette oscillation qui lui fait visiter passé et futur sans jamais perdre de vue la précarité de son existence. La promesse d'une postérité qui permettrait de la prolonger ne s'accorde pas toujours avec les soubassements matérialistes de sa pensée, qui lui font valoriser l'bic et nunc comme lieu de prédilection de l'expérience humaine et poétique. Dans la conclusion du Temple de Mémoire, poème allégorique composé dans les années 1740 (et probable réponse au Temple du gồt de Voltaire), le rêve de postérité ne tient pas devant la logique hédoniste ancrée dans le réel, qu'il défend en convoquant pêle-mêle Carpe diem horatien et Suave mari magno lucrécien:

Qui que tu sois qui m'auras entendu,

Ris, mange, bois, le piège est retendu.

Du dieu moqueur le cri nous importune,

Faisons les sourds: c'est le traitre Neptune,

Qui dans le calme invite à naviguer.

Et toi qui sais plaire, autant qu'obliger,

Dont la belle âme en procédés excelle,

(25) Lettre inédite du poète Piron sur la querelle des Anciens et des Modernes, publiée par P. Verèb dans Alexis Piron défenseur des Modernes, ou un épisode inédit de la Querelle des Anciens et des Modernes, «Revue d'Histoire Littéraire de la France», 1995/2, pp. 282-293.

(26) P. VERÈB, art. cit., p. 285.

(27) Lettre inédite du poète Piron cit., pp. 292-293.

(28) Ibid., p. 290. 
Dont le génie en tout genre étincelle,

Qui fait le bien et le beau tour à tour,

Sans exiger ni gloire, ni retour,

Sans que ton nom, à l'aide du Mercure,

Dans Moreri de se nicher ait cure;

Sans autre vue, après avoir bien fait,

Que le plaisir de t'être satisfait,

De ces vers-ci reçois la dédicace,

Ami Salley, leur morale efficace

De ton esprit a passé dans le mien:

Être, c'est tout: avoir été n'est rien"29.

Ce dernier vers résonne bien sûr avec son épitaphe bien connue, mais plus encore avec les vers qui en concluent la version «longue», moins souvent citée:

[...] Il ne voulut rien être,

Et vécut nul: en quoi certes il fit bien;

Car après tout, bien fou qui se propose,

Venu de rien, et revenant à rien,

D'être en passant ici-bas quelque chose $!^{30}$.

Chez Piron, l'obsession de la gloire côtoie le continuel mépris affiché à son égard: rien que de banal, sans cette fascination pour un rien paradoxalement érigé en objet de désir et marque de l'accomplissement poétique.

Dans un réjouissant conte satirique intitulé La Malle Bosse, Piron s'est moqué des prétentions de son grand ennemi Voltaire, qui lui inspire ici le personnage de Similor. Il le peint en auteur imbu de soi qui s'inquiète de la postérité en ces termes:

Doucement, doucement, notre ami [...] vous ne savez pas, comme un homme de mon état, ce que c'est que d'avoir maille à partir avec la Postérité. S'il est fâcheux, comme vous en convenez, de mourir une fois, vous conviendrez qu'il l'est encore plus d'en mourir deux; et il ne faudrait qu'un placard comme celui-là, sur la tombe d'un illustre, pour le désimmortaliser tout net, ou qui pis est, pour immortaliser ses sottises; car à qui n'arrive-t-il pas d'en faire! ${ }^{31}$.

On relèvera le néologisme qui trouve assurément des échos chez un poète qui a lui-même été «désimmortalisé» (c'est-à-dire reçu à l'Académie Française puis radié du rang des Immortels), qui a connu le succès et a été aspiré dans l'oubli volontaire appliqué à presque tout le théâtre et la poésie de son siècle. La Malle Bosse propose un échantillon de «critique fiction» en imaginant un écrit trouvé par Similor dans une malle de colporteur, le Nouveau supplément du dictionnaire de Moréri daté de 1801, qui anticipe donc sur la fortune des auteurs vivants. Similor commence par en admirer le projet:

Si non vero, bene trovato! bon cadre à jeter de belles vérité au nez des vivants, supposés morts alors! Il ne cessait de se récrier sur la commodité de ce plan, et pour démontrer qu'il était très ingénieux, il dit vingt et vingt fois qu'on le lui avait volés

(29) Le Temple de Mémoire, dans OC, t. VIII, p. 303.

(30) Mon épitaphe, épigramme, OC, t. IX, p. 47.

(31) La Malle Bosse. Nouvelle nuit de Straparole, OC, t. IX, p. 358.

(32) Ibid., p. 366. 
Le texte de ce Supplément visionnaire est écrit en style de dictionnaire, avec choix significatif des entrées:

Tel avantageux de nos jours, qui, pour quelques faibles productions heureuses, en ce siècle de bagatelles, s'érige, dans ses rêves, un trophée chez M. Titon, devait, selon l'esprit de ces omissions affectées, se voir déjà en 1801, placé au rang des noyés ${ }^{33}$.

Au moment où Piron écrit la Malle Bosse, la description du Parnasse français que rêvait d'ériger Titon du Tillet a été publiée, mais son projet est abandonné depuis longtemps: le monument de mémoire est lui-même tombé dans l'oubli et le rêve de s'y voir associé n'en paraît que plus vain. Similor a ensuite une mauvaise surprise, en lisant l'article qui lui est consacré et qui le décrit comme un poète mineur, courtisan et intrigant:

Similor (Matthieu). Écrivain superficiel et fleuri, qui brillait encore vers le milieu du dernier siècle. Ses ouvrages nombreux alors, et dont il ne nous reste que des fragments, durent leur peu de vogue à l'étrange activité qu'il eut, de son vivant, à leur procurer des suffrages. Il sut s'introduire chez les grands et s'insinuer chez les femmes, qui distribuaient alors les honneurs du Parnasse. Il déprimait à demi-mot les bons poètes, exaltait effrontément les mauvais, et soudoyait nombre de prôneurs. Il faisait composer, et composait lui-même ses éloges, que, par des envois anonymes, il faisait ensuite insérer dans les feuilles périodiques, dont la capitale, et les provinces étaient alors infectées: tout ce manège ne le sauva pas d'un grand discrédit, et même de son vivant. Il n'était presque plus mention de lui sur la fin de sa carrière: de là vient qu'on ne sait pas précisément où, quand, ni comment il mourut $[\ldots]^{34}$

C'est très exactement le souci de la gloire qui est dénoncé comme la cause du discrédit des poètes, et c'est leur trop grand désir «d'avoir été» qui les condamne à disparaître des mémoires.

Pour Piron, le moyen d'échapper au fantasme risible d'une postérité à la Titon ou à la Similor sera de «vivre nul», moyen paradoxal d'échapper à l'anéantissement, de faire du néant la matrice de son existence plutôt que le résultat de sa disparition. Piron préconise donc de s'installer dans le présent pour s'y accomplir comme poète, et n'envisage l'avenir que comme le moment où l'on pourra vérifier rétrospectivement la validité de l'expérience hédoniste et poétique.

\section{Le moment et la mémoire}

L'appel au jugement présent et futur est décelable dans sa pièce la plus connue: l'intrigue de La Métromanie s'inscrit dans le cadre très circonstanciel de «l'affaire» Malcrais de la Vigne mais l'anecdote historico-littéraire qui parle aux contemporains est l'occasion de peindre un type universel, le «poète» du sous-titre qui s'adresse aux générations futures:

Qu'on me laisse, à mon gré, n'aspirant qu'à la gloire,

Des titres du Parnasse anoblir ma mémoire,

Et primer dans un art plus au-dessus du droit,

Plus grave, plus sensé, plus noble qu'on ne croit. 
$[\ldots]$

C'en est fait: pour barreau, je choisis le théâtre;

Pour client, la vertu; pour lois, la vérité;

Et pour juges, mon siècle et la postéritée ${ }^{35}$.

Piron joue souvent avec l'idée de vision posthume, qui donnerait accès à la fortune attendant l'homme ou le poète après sa mort. Dans La Malle Bosse, c'est le Nouveau supplément au dictionnaire de Moréri de 1801 qui permet cette projection critique. Une rêverie similaire lui fait entrevoir les circonstances de son «apothéose» ${ }^{36}$ ou considérer tout le bénéfice qu'une grave maladie peut apporter à celui qui se voit, de son vivant, encensé par de trop précoces thuriféraires:

Vivre ainsi, c'est vivre en délices;

C'est de son immortalité

Goûter les heureuses prémices;

Se voir dans la postérité,

Se lire de loin dans l'histoire,

Y savourer déjà sa gloire,

Et bien avant de n'être plus,

Jouir vivant des honneurs dus

Et réservés à sa mémoire ${ }^{37}$.

«Se voir dans la postérité» résume assez bien ce que Piron s'efforce de faire en inventant pour le poète une temporalité bien à lui, qui lui permet de se projeter dans le futur sans perdre pied avec son temps, où «savourer» et «jouir» des honneurs poétiques ont vraiment du sens, où le souci de postérité n'étouffe pas l'appel à «vivre».

On retrouve ici l'ambivalence qui caractérisait la figure du poète chez Piron: l'immortalité est à la fois une belle ambition et une vaste supercherie. Le Temple de Mémoire apparaît régulièrement dans son œuvre, tantôt comme la reprise d'une allégorie à même de traduire ses aspirations profondes, tantôt comme un moyen de railler les honneurs artificiels promis par les Académies. La limite est parfois ténue entre le point de vue satirique et la vision bienveillante. Ainsi voit-on dans l'opéra comique Le Caprice (1724), un poète rêvant d'accéder à la gloire:

Dites, dites plutôt de monter à la gloire,

Et d'aller prendre place au temple de Mémoire!

Aucune formule n'est assez grandiloquente pour décrire son ambition démesurée:

Le beau dessein qui m'anime

Est un dessein magnanime,

D'aller sur la double cime,

Pour vivre au-delà des temps ${ }^{38}$.

(35) La Métromanie cit., acte III, scène 7, pp. 125-126.

(36) Dans L'Apothéose de Binbin, ou Voyage de l'Empyrée.

(37) Lettre à M. Joly de Fleury sur sa convalescence, Euvres complètes [...] par M. R. de Juvigny cit., t. VI, p. 142.

(38) Le Caprice. Opéra comique en un acte, OC, t. IV, scène 11, p. 268. 
Mais le poète glorieux est toujours l'autre face du poète de génie, ce que rappelle Piron en faisant paraître dans ses pièces des personnages de béotiens, qui opposent leur bon sens au désir de gloire poétique. Ainsi l'oncle de Damis dans La Métromanie:

DAMIS

Je veux désormais [...]

Me suffire à moi-même en volant à la gloire,

Et chercher la fortune au temple de Mémoire.

BALIVEAU

Où la vas-tu chercher? Ce temple prétendu

(Pour parler ton jargon) n'est qu'un pays perdu,

Où la nécessité, de travaux consumée,

Au sein du sot orgueil se repaît de fumée ${ }^{39}$.

Ou encore le père du poète dans Le Caprice:

LE PÈRE

Quelles chiennes de visions! Eh! maroufle, songe à gagner ta vie, avant l'immortalité!

LE POÈTE (Air: Vivent les gueux)

Ah! mon âme peu commune

Songe au renom

Plus qu'aux biens de la fortune;

Et mon démon

Porte à la gloire tous mes vœux! $!^{40}$.

Comme on peut le constater, la vaine gloire et le fier honneur du poète peuvent se toucher de près jusqu'à se confondre. «Quand on a l'ambition de travailler pour la postérité, on travaille pour une terrible pratique»: cette grave confidence faite dans une lettre à l'abbé Dumay ${ }^{41}$ n'empêche pas Piron de porter un regard caustique sur tous ceux, lui-même peut-être, qui n'auraient pas les moyens de cette ambition. Le Temple de Mémoire relève de cette forme de satire autocritique. Il y peint un temple que Momus, le dieu de la raillerie, a édifié sur un «un sable très mouvant», avec des matériaux fragiles:

Le frêle en bas, et le solide au faîte:

Ordre, goût, plan, tout dispute au terrain

L'honneur du sceau de sa burlesque main ${ }^{42}$.

À l'appel de Momus invitant les hommes de génie à venir «graver [leurs] noms fameux», une foule accourt, aussitôt dénigrée par Piron:

(39) La Métromanie cit., acte III, scène 7, p. 124.

(40) Le Caprice, OC, t. IV, scène 11, p. 269.

(41) «Quand on a l'ambition de travailler pour la postérité, on travaille pour une terrible pratique. Elle ne se soucie pas des modes. [...] Puisque ma fortune littéraire a reçu des bornes de mon vivant par les bontés de l'Académie, par la piété de M. de Mirepoix et par la justice du roi, étendons les bornes du temps et exerçons nous à mériter quand je ne serai plus là la place que, de mon vivant, je n'ai eu qu'en détrempe. J'ai été élu et exclu par contumace, payons du moins de notre personne à la postérité pour y gagner là mon procès» (Lettre à l'abbé Dumay (20 avril 1754), citée par P. Verèb, cit., p. 285).

(42) Le Temple de Mémoire, OC, t. VIII, p. 298. 
On vole au temple, on s'y porte, on s'y rue,

Chacun se croit l'homme rare et divin,

Et le plus sot, sans faute, est le plus vain ${ }^{43}$.

Le poète renonce «à détailler la foule» de ceux qui essaient par tous les moyens de laisser leurs noms sur les murs ou les colonnes du temple. Pas de postérité pour cette nuée de «moucherons»:

Où courez-vous! insensés que vous êtes!

Quelle vapeur a dérangé vos têtes?

S'écrie en vain le bon sens délaissé:

À vos aïeux appartint le passé,

Que le présent soit votre espoir unique.

Abandonnez l'avenir chimérique

À vos neveux: il ne peut être à vous ${ }^{44}$.

Le mot d'ordre que Piron adresse aux mauvais auteurs en limitant leur espoir au seul présent est proche de celui qu'il s'applique à lui-même, lorsqu'il professe qu'«ÊEtre c'est tout, avoir été n'est rien». C'est de cette lucidité autocritique que son discours sur la vaine gloire tire son originalité et son efficacité. Piron ne s'épargne guère, et se comprend dans la foule des métromanes et des poètes insensés. L'image de Pégase fixe dans son œuvre le travail de désillusion qu'il s'impose. Dans Le Temple de Mémoire, le cheval du Parnasse est l'occasion d'un confiteor amusé et contrit de la part d'un Piron que sa satire finit par atteindre:

Jusqu'au pinacle enfin quelqu'autre vole,

À la faveur du quadrupède ailé,

Sur qui je n'ai que trop caracolée ${ }^{45}$.

Dans L'Apothéose de Binbin, l'apostrophe à Pégase honore la poésie et humilie le poète dans un même mouvement lyrique et satirique:

Vous voilà donc, noble sang de Méduse,

Dis-je au coursier de l'immortalité!

Sur vous cent fois je me suis cru monté,

Quand le jouet d'une folâtre muse,

Comme un nigaud qu'aisément on abuse,

Sur chevillard je n'étais que plantée ${ }^{46}$.

La gloire momentanée que l'on obtient dans les salons est aussi vaine que l'appel illusoire à la postérité. Le moment et la mémoire sont deux vanités auxquelles Piron tâche de substituer le présent, une durée où le plaisir d'être poète puisse prétendre à se survivre.

L'Apothéose de Binbin est peut-être le texte qui illustre le mieux cet équilibre instable entre élégante indifférence de la gloire et opiniâtre désir tourné vers la posté-

(43) Ibid., p. 299.

(44) Ibid., p. 301.

(45) Ibid., p. 302.

(46) L'Apothéose de Binbin, OC, t. VIII, p. 335. 
rité. Dans ce poème composé sur la fin de sa vie, Piron décrit son apothéose de poète sous son nom familier de Binbin, et confond ainsi les deux tentations de prendre au sérieux ou de tourner en dérision la vocation poétique. Imaginant que son âme se détache de son corps, Piron observe de loin la terre et s'étonne de la voir si minuscule:

Le voilà donc ce globe terrien,

Dis-je en riant, qu'on s'imagine immense!

Où du néant sorti pour être rien,

Né d'une femme et d'un homme de bien,

Faute de nom, de titres, de chevance,

Et d'y pourvoir n'ayant aucun moyen,

J'arrivai nu et naquis sans naissance ${ }^{47}$.

Le rire de n'avoir été rien laisse place cependant à la fierté de n'être pas l'une des «fausse[s] comète[s] $\rrbracket^{48}$ qui traversent le ciel, et c'est avec assurance qu'à l'invitation de Pégase il gagne sa place dans l'empyrée:

Il dit: mon âme, à ces mots rassurée

Sur quelque bruit que je laisse après moi,

Mon adieu fait, je gagnai l'empyrée,

Où me voici brillant, tranquille et coi ${ }^{49}$.

La tranquille immortalité du poète n'est pas sans paradoxe, puisqu'il lui faut dire son mutisme, chanter qu'il se tient coi, que la modestie avec laquelle il évoque le «bruit» qu'il laisse après lui est contredite par le «brillant» dont il se pare. On retiendra surtout que c'est encore au présent que Piron se présente: il ne fut rien, pas même académicien, et le voici, toujours poète, léguant à ceux qui viennent la conscience exigeante de l'impermanence de toutes choses pour pouvoir élever une voix digne d'être entendue, capable de dénoncer la vanité du désir de postérité et d'en revendiquer tous les droits. 